\title{
Relationship between Gait, Static Balance, and Pelvic Inclination in Patients with Chronic Stroke
}

\author{
Yu-Won Choe, PT, MS • Kyu-Ryeong Kim, PT, MS • Myoung-Kwon Kim, PT, PhD ${ }^{1 \dagger}$ \\ Department of Rehabilitation Sciences, Graduate School, Daegu University, \\ ${ }^{1}$ Department of Physical Therapy, College of Rehabilitation Sciences, Daegu University
}

Received: October 29, 2020 / Revised: November 16, 2020 / Accepted: December 12, 2020

(C) 2021 J Korean Soc Phys Med

\section{| Abstract |}

PURPOSE: This study examined the correlations between gait, static balance, and pelvic inclination in patients with chronic stroke.

METHODS: Twenty-two chronic stroke patients were included in this study. The subjects participated in gait, static balance, and pelvic inclination tests. In the gait measurement, the cadence and gait velocity were measured, and the average of three trials was calculated and recorded. The static balance was measured using a force platform. The data was captured for ten seconds, and five successful trials were recorded. Pelvic inclination in the sagittal plane was measured using a palpation meter. For data processing, a KolmogorovSmirnov test was used to determine the type of distribution for all variables. Pearson's correlation coefficient was used for correlation analysis. The correlations among the gait, static balance, and pelvic inclination was calculated. The level of

$\uparrow$ Corresponding Author : Myoung-Kwon Kim skybird-98@hanmail.net, https://orcid.org/0000-0002-7251-6108 This is an Open Access article distributed under the terms of the Creative Commons Attribution Non-Commercial License (http://creativecommons.org/licenses/by-nc/3.0) which permits unrestricted non-commercial use, distribution, and reproduction in any medium, provided the original work is properly cited. significance was .05 .

RESULTS: Significant negative correlations were observed between the gait variables (cadence, velocity) and static balance variables (COP path length, COP average velocity, and $95 \%$ confidence ellipse area) $(p<.05)$. On the other hand, there was no significant correlation between pelvic inclination and gait or between the pelvic inclination and static balance variables.

CONCLUSION: Significant correlations were observed between the gait function and static balance. On the other hand, there were no significant correlations between the pelvic inclination and gait and static balance. These results suggest that the pelvic inclination is not an important consideration for increasing the gait function and static balance.

Key Words: Balance, Gait, Pelvic inclination

\section{Introduction}

Stroke patients have paralysis, and most complain of difficulty in walking [1]. The common feature of walking after a stroke includes decreased gait speed. Intervention of physical therapy for stroke patients includes an aim to increase walking speed [2]. Stroke patients have also 
increased the body sway of the center of pressure because of the decreased balance function and distorted standing posture [3,4]. Postural instability and poor balance function decrease the gait ability, which causes difficulties in daily life [3]. Furthermore, abnormal pelvic alignment and asymmetrical weight bearing onto the lower limbs are common findings observed in standing and walking after stroke $[5,6]$. The altered pelvic alignment results from reduced hip muscular control or poor trunk-pelvis dissociation [1,7], and abnormal pelvic movement, such as anterior pelvic tilt, occurs in patients with stroke $[1,8]$.

Kong et al. examined 58 people with chronic stroke to check the correlation between the gait and balance and pelvic displacement [3]. This study used the stability index and weight distribution index for the balance test and used a 10-Meter Walk Test and Figure-of- 8 Walk Test for the gait test [3]. They reported that the increased pelvic displacement results in a decrease in gait velocity and balance ability in stroke patients [3]. In addition, this study did not focus on the cadence and gait velocity, which is the most important variable when evaluating the gait function, and did not address the shift of the center of pressure that should be checked when evaluating the static balance ability [3]. Another study also confirmed the correlation between the pelvic tilt and gait, balance in chronic stroke patients [1]. This study evaluated the balance function by measuring the moving distance and area of the center of pressure and tested the gait function by measuring the cadence and gait velocity [1]. The study showed a negative correlation between the anterior pelvic tilt and the gait ability, including gait velocity and step length in chronic patients [1]. On the other hand, there was no significant correlation between balance function and pelvic tilt [1]. Karthikbabu et al. reported the relationship between abnormal asymmetric weight-bearing and the pelvic alignment in patients with chronic stroke [5]. They reported that the asymmetrical weight-bearing of chronic stroke is affected more by the lateral pelvic alignment than the anterior-posterior alignment [5].

Several studies examined the correlation between gait and balance, pelvic inclination, but the results were conflicting $[1,3,5]$. Therefore, this study examined whether gait is correlated with the balance function and pelvic inclination in chronic stroke. The hypothesis of the present study was that there would be a correlation between gait function and static balance, but there will not be significant correlations between the pelvic inclination and gait, and static balance.

\section{Methods}

\section{Participants}

Twenty-two chronic stroke patients who agreed to participate in this experiment were included in the study. Before the start of the study, all subjects understood its content and signed an informed consent form. This study complied with the ethical standards of the declaration of Helsinki and was approved by the ethical committee of Daegu University (1040621-201909-HR-027-02). The subjects were required to meet the following inclusion criteria: 1) the first onset of stroke and onset duration of more than six months $[9,10], 2)$ no cognitive impairment $(>24$ points on the mini-mental state examination-Korean version $[9,10], 3)$ the subjects could understand and follow the therapist's directions [10], 4) ability to stand and walk 10 meters independently without supervision [9], and 5) no other neurological or orthopedic disorders $[9,10]$.

\section{Experimental procedures}

This study was a cross-sectional design. The subjects participated in gait, static balance, and pelvic inclination tests. The order of tests was as follows: 1) pelvic inclination, 2) static balance, and 3) gait function. The participants had a five-minute break between measurements. All tests of the participant were performed in a single day. Fig. 1 presents a flowchart of the study. 


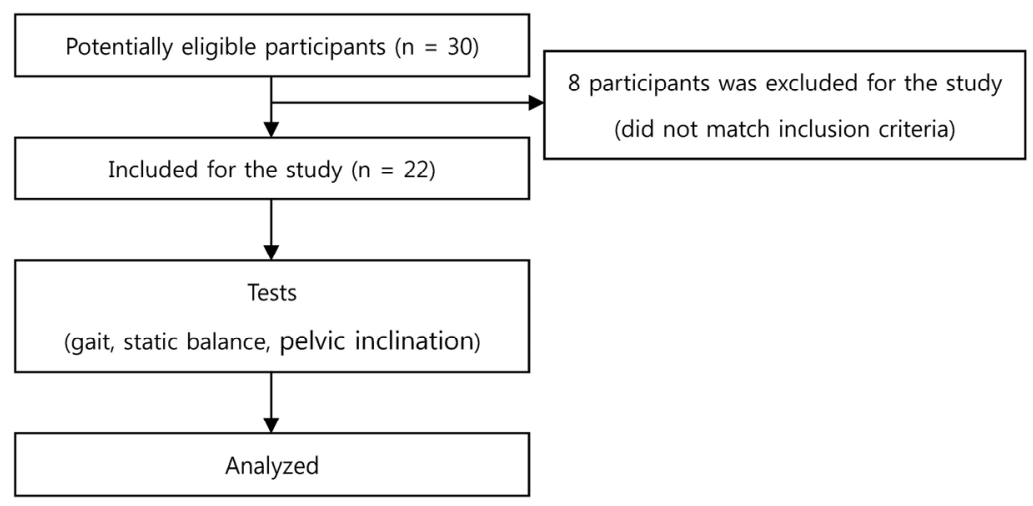

Fig. 1. Study flowchart

\section{Measurement Items}

\section{1) Gait Function}

The GAITRite (GaitRite, CIR Systems Inc., USA) system evaluates the temporal parameters and spatial parameters [11]. The GAITRite mat exhibits excellent reliability of walking speed and cadence (ICCs between .82 and .92) in older subjects [12]. In this study, the cadence and gait velocity were measured. For the measurements, the subjects were instructed to stand $3 \mathrm{~m}$ away from the electronic carpet and walk across the carpet at a comfortable walking speed, stopping after walking $3 \mathrm{~m}$ past the electronic carpet [11]. The measurements were repeated three times with a three-minute break between the measurements to minimize the bias caused by muscle fatigue [11]. The average of the three measurements was calculated and recorded [11].

\section{2) Static Balance}

The force platform (FDM SX, Zebris, Germany) was used to measure the static balance. This force platform was a $55 \times 40 \mathrm{~cm}$ plate and sensor surface, a 40 × 30 $\mathrm{cm}$ in size with a 1,920 pressure sensor. The sampling rate of the device was $120 \mathrm{~Hz}$. Previous studies reported a good ICC of $>.90$ for this system $[13,14]$. The force platform system measured the center of pressure (COP) sway path length and the average COP sway velocity. A longer COP sway path length or higher COP sway velocity indicated inferior static standing balance performance [15]. The confidence ellipse area, which is the standard deviation of the vector of COPs [14], was also measured. In this test, all subjects stood on the platform barefoot with their hands next to their bodies and stared at the front wall $[14,16]$. The location of the feet was marked to ensure that they were positioned in the same place during the reassessment [16]. For testing with their eyes open, the subjects were asked to stare at a $15 \mathrm{~cm}$ diameter dot placed $3 \mathrm{~m}$ ahead [16]. The data was captured for ten seconds, and five successful trials were recorded with a thirty-second rest interval [14]. The mean value of five successful trials was used for data processing.

\section{3) Pelvic Inclination}

Pelvic inclination was measured using a palpation meter (PALM; Performance Attainment Associates, St. Paul, MN, USA) by one inspector. The PALM is composed of an inclinometer, two caliper arms. This equipment is a tool that calculates the difference in height between landmarks. The PALM is a clinically valid, inexpensive, and reliable tool. The intra-tester and inter-tester reliability of the sagittal plane measures was high (.98 and .89 , respectively), and the reliability of the sagittal plane measures was high 
(.89) [17]. The subjects wore non-restrictive clothing and removed their shoes during the measurements. They spread their feet (approximately $1012 \mathrm{~cm}$ ) and stood in a relaxed posture. They stood in an upright position, leaning the anterior aspect of the thighs against a stabilizing table. The investigator palpated the prominence of the ipsilateral ASIS and PSIS and marked them with a pen. The sagittal plane rotation of the innominate bone was measured using the caliper tips of the PALM in contact with the ipsilateral ASIS and PSIS [18,19].

Table 1. General Characteristics of the Subjects

\begin{tabular}{lc}
\hline & $\mathrm{N}=22$ \\
\hline Age (years) & $65.727 \pm 6.188$ \\
Time Since Onset (month) & $17.275 \pm 5.238$ \\
Height (cm) & $162.045 \pm 7.201$ \\
Weight (kg) & $62.818 \pm 9.574$ \\
Gender (male/female) & $11 / 11$ \\
Paretic Side (right/left) & $10 / 12$ \\
Type of Stroke (ischemia/hemorrhage) & $12 / 10$ \\
\hline Mean \pm SD &
\end{tabular}

\section{Data Processing}

SPSS 20.0 software (SPSS Inc., Chicago, IL) was used for all statistical analyses. A KolmogorovSmirnov test was used to determine the type of distribution for all variables. The Pearson's correlation coefficient was used for correlation analysis. The correlations among the gait, static balance, and pelvic inclination were calculated. The level of significance was .05 .

\section{Results}

Twenty-two subjects participated in this study. Table 1 lists the general characteristics of the subjects (Table 1). Table 2 presents the correlations between the gait and static balance and pelvic inclinations (Table 2). Significant negative correlations were observed between the gait variables (cadence, velocity) and static balance variables (COP path length, COP average velocity, and 95\% confidence ellipse area) $(p<.05)$. On the other hand, there were no significant correlations between pelvic inclination and gait. There was no significant correlation between the

Table 2. Correlations Among the Variables

\begin{tabular}{|c|c|c|c|c|c|c|c|}
\hline & $\mathrm{n}=22$ & $\begin{array}{l}\text { Velocity } \\
(\mathrm{cm} / \mathrm{s})\end{array}$ & $\begin{array}{c}95 \% \text { CEA } \\
\left(\mathrm{mm}^{2}\right)\end{array}$ & $\begin{array}{c}\text { COP PL } \\
(\mathrm{mm})\end{array}$ & $\begin{array}{l}\text { COP AV } \\
(\mathrm{mm} / \mathrm{sec})\end{array}$ & $\begin{array}{c}\text { AS PI } \\
\text { (degree) }\end{array}$ & $\begin{array}{l}\text { LAS PI } \\
\text { (degree) }\end{array}$ \\
\hline $\begin{array}{c}\text { Cadence } \\
\text { (steps/min) }\end{array}$ & $58.068 \pm 15.613$ & $.948 * *$ & $-.894 * *$ & $-.563^{* *}$ & $-.564 * *$ & -.253 & -.150 \\
\hline $\begin{array}{l}\text { Velocity } \\
(\mathrm{cm} / \mathrm{s})\end{array}$ & $23.195 \pm 9.980$ & & $-.900 * *$ & $-.603 * *$ & $-.604 * *$ & -.289 & -.195 \\
\hline $\begin{array}{c}95 \% \text { CEA } \\
\left(\mathrm{mm}^{2}\right)\end{array}$ & $1265.772 \pm 729.684$ & & & $.675^{* *}$ & $.677 * *$ & .322 & .229 \\
\hline $\begin{array}{l}\text { COP PL } \\
(\mathrm{mm})\end{array}$ & $203.636 \pm 84.476$ & & & & $.999 * *$ & .177 & .088 \\
\hline $\begin{array}{l}\text { COP AV } \\
(\mathrm{mm} / \mathrm{sec})\end{array}$ & $20.272 \pm 8.435$ & & & & & .181 & .091 \\
\hline $\begin{array}{c}\text { AS PI } \\
\text { (degree) }\end{array}$ & $11.140 \pm 2.980$ & & & & & & $.952 * *$ \\
\hline $\begin{array}{l}\text { LAS PI } \\
\text { (degree) }\end{array}$ & $10.618 \pm 2.315$ & & & & & & \\
\hline
\end{tabular}

Mean \pm SD, 95\% CEA: 95\% confidence ellipse area, COP PL: center of pressure path length, COP AV: center of average pressure velocity, AS PI: pelvic inclination on the affected side, LAS PI: pelvic inclination on the less affected side, $*^{*}<.05, *^{*} p<.01$ 
pelvic inclination and static balance variables.

\section{Discussion}

In this study, significant negative correlations were observed between gait variables (cadence, velocity) and static balance variables (COP path length, COP average velocity, and $95 \%$ confidence ellipse area). These results suggest that cadence and gait velocity increase if the body sway decreases. The results are consistent with an earlier study. Previous studies revealed a significant relationship between the spatiotemporal gait function and standing balance function and reported that improved balance might enhance the gait function [20,21]. Another study showed that the gait speed and cadence are factors related to the balance performance [22]. One study explained why increasing the balance function could lead to a better gait function because patients with a better balance function during gait could walk within a narrow BOS, indicating their ability to shift their COG in all A-P and M-L directions without losing balance [21]. Another study reported that impaired paretic limb for balance control might contribute to the asymmetric gait post-stroke. They suggested that intervention to increase the contribution of the paretic limb to the standing balance control might increase the symmetric of walking post-stroke [23].

In this study, there were no significant correlations between the pelvic inclinations, gait, and static balance variables. Significant positive correlations were observed between the pelvic inclinations on the affected side and the less affected side. These findings are inconsistent with those reported elsewhere. A previous study reported a positive correlation between the pelvic displacement, weight distribution index $(\mathrm{r}=.38)$, and the 10-meter walk test $(r=.41)$ [3]. These results mean increased pelvic displacement causes a decreased balance ability and gait speed in stroke patients [3]. Another study reported a significant negative correlation between the gait velocity and pelvic anterior tilt $(\mathrm{r}=-.61)$ [1]. The inconsistency was attributed to a difference in measurement tools or number of subjects between studies. Another possible explanation is that the stroke patients participated in this study had various postures, such as kyphosis, lordosis, or pelvic displacement, even before the stroke. In addition, a previous study reported that weight bearing with chronic stroke patients, which is important for gait and balance, is affected more by the lateral pelvic alignment than anterior-posterior alignment [5]. Therefore, anterior pelvic inclination could not affect the gait and balance function.

This study had several limitations. First, this study was a cross-sectional design and identified the correlations between the gait and static balance, pelvic inclination. Second, the sample size was small. Given these limitations, an additional longitudinal study will be needed.

\section{Conclusion}

Significant correlations were observed between the gait function and static balance. On the other hand, there were no significant correlations between the pelvic inclination and gait, and static balance. These results suggest that the pelvic inclination is not an important consideration for increasing the gait function and static balance.

\section{References}

[1] Kim MK, Kim SG, Shin YJ, et al. The relationship between anterior pelvic tilt and gait, balance in patient with chronic stroke. J Phys Ther Sci. 2018;30(1):27-30.

[2] Fujita K, Hori H, Kobayashi Y. Contribution of muscle activity at different gait phases for improving walking performance in chronic stroke patients with hemiparesis. Journal of physical therapy science. 2018;30(11):13815.

[3] Kong SW, Jeong YW, Kim JY. Correlation between balance and gait according to pelvic displacement in stroke patients. J Phys Ther Sci. 2015;27(7):2171-4. 
[4] Hugues A, Di Marco J, Janiaud P, et al. Efficiency of physical therapy on postural imbalance after stroke: study protocol for a systematic review and meta-analysis. BMJ open. 2017;7(1):e013348.

[5] Karthikbabu S, Chakrapani M, Ganesan S, et al. Relationship between Pelvic Alignment and Weight-bearing Asymmetry in Community-dwelling Chronic Stroke Survivors. J Neurosci Rural Pract. 2016;7(Suppl 1):S3740.

[6] Tyson SF. Trunk kinematics in hemiplegic gait and the effect of walking aids. Clin Rehabil. 1999;13(4):295-300.

[7] Patterson KK, Parafianowicz I, Danells CJ, et al. Gait asymmetry in community-ambulating stroke survivors. Arch Phys Med Rehabil. 2008;89:30410.

[8] Kim HS, Chung SC, Choi MH, et al. Primary and secondary gait deviations of stroke survivors and their association with gait performance. J Phys Ther Sci. 2016:28;263440

[9] Kim GM, Oh DW. Neck proprioceptive training for balance function in patients with chronic poststroke hemiparesis: a case series. J Phys Ther Sci. 2014;26(10): 1657-9.

[10] Lee JH, Choi JD. The effects of upper extremity task training with symmetric abdominal muscle contraction on trunk stability and balance in chronic stroke patients. J Phys Ther Sci. 2017;29(3):495-7.

[11] Choi W, Han D, Kim J, et al. Whole-Body Vibration Combined with Treadmill Training Improves Walking Performance in Post-Stroke Patients: A Randomized Controlled Trial. Med Sci Monit. 2017;23:4918-25.

[12] Menz HB, Latt MD, Tiedemann A, et al. Reliability of the GAITRite walkway system for the quantification of temporo-spatial parameters of gait in young and older people. Gait Posture. 2004;20(1):20-5.

[13] Nakhaee Z, Rahimi A, Abaee M, et al. The relationship between the height of the medial longitudinal arch (MLA) and the ankle and knee injuries in professional runners.
Foot. 2008;18(2):84-90.

[14] Soltani N, Rahimi A, Naimi SS, et al. Studying the Balance of the Coper and Non-Coper ACL-Deficient Knee Subjects. Asian J Sports Med. 2014;5(2):91-8.

[15] Wong T, Ma A, Liu K, et al. Balance control, agility, eye-hand coordination, and sport performance of amateur badminton players: A cross-sectional study. Medicine. 2019;98(2):e14134.

[16] In T, Lee K, Song C. Virtual Reality Reflection Therapy Improves Balance and Gait in Patients with Chronic Stroke: Randomized Controlled Trials. Med Sci Monit. 2016;22:4046-53.

[17] Hagins M, Brown M, Cook C, et al. Intratester and intertester reliability of the palpation meter (PALM) in measuring pelvic position. J Man Manip Ther. 1998; 6(3):130-6.

[18] Gnat R, Saulicz E, Biały M, et al. Does pelvic asymmetry always mean pathology? Analysis of mechanical factors leading to the asymmetry. J Hum Kinet. 2009;21(1):23-32.

[19] Lee JH, Yoo WG, Gak HB. The immediate effect of anterior pelvic tilt taping on pelvic inclination. J Phys Ther Sci. 2011;23(2):201-3.

[20] Lewek MD, Bradley CE, Wutzke CJ, et al. The relationship between spatiotemporal gait asymmetry and balance in individuals with chronic stroke. J Appl Biomech. 2014; 30:31-6.

[21] An CM, Son YL, Park YH, et al. Relationship between dynamic balance and spatiotemporal gait symmetry in hemiplegic patients with chronic stroke. Hong Kong Physiother J. 2017;37:19-24.

[22] Obembe AO, Olaogun MO, Adedoyin R. Gait and balance performance of stroke survivors in South-Western Nigeria-A cross-sectional study. Pan Afr Med J. 2014;17(Suppl 1).

[23] Hendrickson J, Patterson KK, Inness EL, et al. Relationship between asymmetry of quiet standing balance control and walking post-stroke. Gait Posture. 2014;39(1)177-81. 